\title{
RANDOM SEMILINEAR EVOLUTION EQUATIONS IN BANACH SPACES
}

\author{
DIMITRIOS KRAVVARITIS
}

(Communicated by Palle E. T. Jorgensen)

\begin{abstract}
In this paper we prove the existence of mild solutions for random, semilinear evolution equations involving a random, linear, unbounded $m$-dissipative operator and a random single valued or multivalued perturbation. Finally, an application to a random semilinear partial differential equation is given.
\end{abstract}

\section{INTRODUCTION}

The theory of random differential equations has attracted the attention of many authors (e.g., Kampé de Feriet [7], Becus [3], Itoh [5, 6], Papageorgiou [9, 10], Avgerinos and Papageorgiou [1] and their references). This interest is due to the fact that there are many applications of this theory to various applied fields such as control theory, statistics, biological sciences, and others. For a discussion of such applications one may consult the books of Bharucha-Reid [4] and Soong [16].

In this paper we present two existence results for random semilinear evolution equations associated with linear, unbounded $m$-dissipative operators in Banach spaces. Deterministic equations of this form were studied by Pazy [14], Vrabie [18], and Pavel [12].

Let $(\Omega, \Sigma, \mu)$ be a complete $\sigma$-finite measure space and $X$ a separable Banach space. $X_{w}$ is the space $X$ endowed with the weak topology. By $P_{f(c)}(X)$ we will denote the nonempty, closed(convex) subsets of $X$. A multifunction $F: \Omega \rightarrow P_{f}(X)$ is said to be measurable if it satisfies one of the following equivalent conditions:

(i) for every $x \in X, \omega \rightarrow d(x, F(\omega))=\inf \{\|x-z\|, z \in F(\omega)\}$ is measurable.

Received by the editors April 23, 1990.

1980 Mathematics Subject Classification (1985 Revision). Primary 47H06, 47H15.

Key words and phrases. $m$-dissipative, mild solution, measurable multifunction, semigroup of contractions, random equations.

This work was done while the author was visiting the University of Kiel, West Germany. The author would like to thank Professor H. König for his invitation and hospitality. 
(ii) there exist $f_{n}: \Omega \rightarrow X, n \geq 1$, measurable functions such that for all $\omega \in \Omega$

$$
F(\omega)=\operatorname{cl}\left\{f_{n}(\omega)\right\}_{n \geq 1} \quad \text { (Castaing's representation). }
$$

(iii) $\operatorname{Gr} F=\{(\omega, x) \in \Omega \times X: x \in F(\omega)\} \in \Sigma \times B(X)$, where $B(X)$ is the Borel $\sigma$-field of $X$ (graph measurability).

By $S_{F}^{p}(1 \leq p \leq \infty)$ we will denote the set of all selectors of $F(\cdot)$ that belong to the Lebesgue-Bochner space $L^{p}(\Omega, X)$, that is, $S_{F}^{p}=\left\{f \in L^{p}(\Omega, X): f(\omega) \in\right.$ $F(\omega) \mu$-a.e. $\}$.

Let $A: D(A) \subset X \rightarrow X$ be a linear, unbounded, $m$-dissipative operator. It is known (e.g. [2]) that $A$ generates a semigroup $\{S(t), t \geq 0\}$ of contractions on $X$ which is given through the Grandall-Liggett exponential formula

$$
S(t) x=\lim _{n \rightarrow \infty}\left(I-\frac{t}{n} A\right)^{-n} x .
$$

Let $T=[0, b]$ a closed, bounded interval in $\mathbb{R}^{+}$. Consider the following initial value problem

$$
\begin{aligned}
& \dot{x}(t)=A x(t)+f(t), \quad t \in T, \\
& x(0)=x_{0},
\end{aligned}
$$

where $f \in L^{1}(T, X)$ and $x_{0} \in X$. A continuous function $x: T \rightarrow X$ is a mild solution of (1) if

$$
x(t)=S(t) x_{0}+\int_{0}^{t} S(t-s) f(s) d s \quad \text { for all } t \in T .
$$

It is known [13] that (1) has a unique mild solution. Let $D$ be a subset of $X$. A mapping $T: \Omega \times D \rightarrow X$ is said to be random, if for each $x \in D, T(\cdot) x: \Omega \rightarrow X$ is measurable. A random operator $T$ is said to be linear(continuous, etc.), if for any $\omega \in \Omega, T(\omega): D \rightarrow X$ is linear(continuous, etc.).

Suppose $Y, Z$ are topological spaces. A multifunction $F: Y \rightarrow 2^{Z} \backslash\{\varnothing\}$ is said to be upper semicontinuous (u.s.c.) (resp. lower semicontinuous (1.s.c)) if for any $U \subset Z$ nonempty open $F^{+}(U)=\{x \in X: F(x) \subset U\}$ (resp. $\left.F^{-}(U)=\{x \in X: F(x) \cap U \neq \varnothing\}\right)$ is open in $X$. Also $F(\cdot)$ is continuous if it is both u.s.c. and l.s.c. Furthermore, we say that $F$ is closed if $\mathrm{Gr} F=$ $\{(y, z) \in Y \times Z: z \in F(y)\}$ is closed in $Y \times Z$.

\section{THE MAIN RESUlTS}

Let $(\Omega, \Sigma, \mu)$ be a complete $\sigma$-finite measure space, $X$ a reflexive, separable Banach space, and $D$ a dense linear subspace of $X$. We consider the following random evolution equation

$$
\begin{aligned}
& \dot{x}(\omega, t)=A(\omega) x(\omega, t)+f(\omega, t), \\
& x(\omega, 0)=x_{0}(\omega),
\end{aligned}
$$

where $A: \Omega \times D \rightarrow X$ is a random, linear $m$-dissipative operator and $f$ a random perturbation. By a random mild solution of (2) we understand a mapping 
$x: \Omega \times T \rightarrow X$ such that for all $t \in T, x(\cdot, t)$ is measurable and for each $\omega \in \Omega, x(\omega, \cdot)$ is a mild solution of $(2)$.

Theorem 2.1. Let $A: \Omega \times D \rightarrow X$ be a random, linear m-dissipative operator and $f: \Omega \times T \rightarrow X$ a measurable mapping such that for each $\omega \in \Omega, f(\omega, \cdot) \in$ $L^{1}(T, X)$. If $x_{0}: \Omega \rightarrow X$ is measurable, then (2) admits a unique random mild solution.

Proof. For each $\omega \in \Omega$, we know (see Pavel [13, p. 129]) that the corresponding deterministic problem has a unique mild solution

$$
x(\omega, t)=S(\omega, t) x_{0}(\omega)+\int_{0}^{t} S(\omega, t-s) f(\omega, s) d s, \quad t \in T .
$$

By the Grandall-Liggett formula we have

$$
S(\omega, t) x=\lim _{n \rightarrow \infty}\left(I-\frac{t}{n} A(\omega)\right)^{-n} x .
$$

From Proposition of [8] we know that $\omega \rightarrow(I-t A(\omega) / n)^{-1} x$ is measurable which implies that $\omega \rightarrow S(\omega, t) x$ is measurable. Using the continuity properties of the semigroup $S(\omega, \cdot)$ and our hypotheses we get that the mappings $\omega \rightarrow S(\omega, t) x_{0}(\omega)$ and $(\omega, s) \rightarrow S(\omega, t-s) f(\omega, s)$ are measurable. By Fubini's theorem $\omega \rightarrow \int_{0}^{t} S(\omega, t-s) f(\omega, s) d s$ is measurable. It then follows that $\omega \rightarrow x(\omega, t)$ is measurable.

Now, consider the following random multivalued evolution equation:

$$
\begin{aligned}
& \dot{x}(\omega, t) \in A(\omega) x(\omega, t)+F(\omega, t, x(\omega, t)), \\
& x(\omega, 0)=x_{0}(\omega),
\end{aligned}
$$

where $A: \Omega \times D \rightarrow X$ is a random, linear $m$-dissipative operator and $F$ is a random multivalued perturbation. By a random mild solution of (3) we understand a mapping $x: \Omega \times T \rightarrow X$ such that for each $t \in T, x(\cdot, t)$ is measurable and for each $\omega \in \Omega, x(\omega, \cdot)$ is a mild solution of

$$
\begin{aligned}
& \dot{x}(\omega, t)=A(\omega) x(\omega, t)+f(\omega, t) \\
& x(\omega, 0)=x_{0}(\omega)
\end{aligned}
$$

for some $f(\omega, \cdot) \in S_{F(\omega, \cdot, x(\omega, \cdot))}^{1}$.

Theorem 2.2. Let $A: \Omega \times D \rightarrow X$ be a random, linear m-dissipative operator such that for each $\omega \in \Omega, A(\omega)$ generates a semigroup $S(\omega, t)$ which is compact for $t>0$. Let $F: \Omega \times T \times X \rightarrow P_{f_{c}}(X)$ be a multifunction such that

(i) $(\omega, t, x) \rightarrow F(\omega, t, x)$ is measurable;

(ii) for every $(\omega, t) \in \Omega \times T$, the multifunction $x \rightarrow F(\omega, t, x)$ is closed in $X \times X_{w}$; and

(iii) $|F(\omega, t, x)|=\sup \{\|z\|: z \in F(\omega, t, x)\} \leq a(\omega, t)+b(\omega, t)\|x\|$ a.e. for all $\omega \in \Omega$, where $a(\cdot, \cdot), b(\cdot, \cdot)$ are jointly measurable and for all $\omega \in \Omega, a(\omega, \cdot), b(\omega, \cdot) \in L^{1}\left(T, \mathbb{R}^{+}\right)$.

If $x_{0}: \Omega \rightarrow X$ is measurable then (3) admits a random mild solution. 
Proof. First, let us determine an a priori bound for the solutions of (3). Let $x(\cdot, \cdot)$ be a random mild solution of $(3)$. Then

$$
x(\omega, t)=S(\omega, t) x_{0}(\omega)+\int_{0}^{t} S(\omega, t-s) h(\omega, s) d s
$$

for some $h(\omega, \cdot) \in S_{F(\omega, \cdot, x(\omega, \cdot))}^{1}$. Since $S(\omega, t)$ is a semigroup of contractions, it follows that

$$
\|x(\omega, t)\| \leq\left\|x_{0}(\omega)\right\|+\int_{0}^{t}\|h(\omega, s)\| d s .
$$

Using the growth hypothesis of $F$ we get

$$
\begin{aligned}
\|x(\omega, t)\| & \leq\left\|x_{0}(\omega)\right\|+\int_{0}^{t}(a(\omega, s)+b(\omega, s)\|x(\omega, s)\|) d s \\
& \leq\left\|x_{0}(\omega)\right\|+\|a(\omega, \cdot)\|_{1}+\int_{0}^{t} b(\omega, s)\|x(\omega, s)\| d s .
\end{aligned}
$$

Invoking the Gronwall inequality we conclude that

$$
\|x(\omega, t)\| \leq k(\omega) \exp \|b(\omega, \cdot)\|_{1}=M(\omega),
$$

where $k(\omega)=\left\|x_{0}(\omega)\right\|+\|a(\omega, \cdot)\|_{1}$.

Now, we consider the multifunction $\widehat{F}: \Omega \times T \times X \rightarrow P_{f c}(X)$ defined by

$$
\widehat{F}(\omega, t, x)= \begin{cases}F(\omega, t, x), & \text { if }\|x\| \leq M(\omega) \\ F(\omega, t, M(\omega) x /\|x\|), & \text { if }\|x\|>M(\omega) .\end{cases}
$$

From this definition we see that $\widehat{F}(\omega, t, x)=F(\omega, t, p(\omega, x))$, where, for every $\omega \in \Omega, p(\omega, \cdot)$ is the $M(\omega)$-radial retraction. Note that $p(\omega, x)$ is measurable in $\omega$ and continuous in $x$. Hence $(\omega, t, x) \rightarrow \widehat{F}(\omega, t, x)$ is measurable and by hypothesis (ii), $x \rightarrow \widehat{F}(\omega, t, x)$ is closed in $X \times X_{w}$. We have

$$
|\widehat{F}(\omega, t, x)| \leq a(\omega, t)+M(\omega) b(\omega, t)=\gamma(\omega, t) .
$$

Clearly $\gamma(\cdot, \cdot)$ is jointly measurable and for all $\omega \in \Omega, \gamma(\omega, \cdot) \in L^{1}\left(T, \mathbb{R}^{+}\right)$. Consider the set

$$
B(\omega)=\left\{h \in L^{1}(T, X):\|h(t)\| \leq \gamma(\omega, t) \text { a.e. }\right\} .
$$

We assert that $\omega \rightarrow B(\omega)$ is a measurable multifunction. To see this let $H(\omega, t)=\{x \in X:\|x\| \leq \gamma(\omega, t)\}$. Since $\gamma(\cdot, \cdot)$ is jointly measurable then so is the multifunction $(\omega, t) \rightarrow H(\omega, t)$. Now, for every $g \in L^{1}(T, X)$ we have

$$
\begin{aligned}
d(g, B(\omega)) & =\inf \left\{\|g-h\|_{1}, h \in B(\omega)\right\} \\
& =\inf \left\{\int_{0}^{b}\|g(s)-h(s)\| d s, h \in B(\omega)\right\} \\
& =\int_{0}^{b} \inf \{\|g(s)-u\|, u \in H(\omega, s)\} d s \\
& =\int_{0}^{b} d(g(s), H(\omega, s)) d s .
\end{aligned}
$$


From Fubini's theorem we get that $\omega \rightarrow d(g, B(\omega))$ is measurable, which implies that $\omega \rightarrow B(\omega)$ is measurable.

Now, let $r: \Omega \times L^{1}(T, X) \rightarrow C(T, X)$ be the map that to each $(\omega, h) \in$ $\Omega \times L^{1}(T, X)$ assigns the unique mild solution of

$$
\begin{aligned}
\dot{x}(t) & =A(\omega) x(t)+h(t) \\
x(0) & =x_{0}(\omega) .
\end{aligned}
$$

Fix $h \in L^{1}(T, X)$. From Theorem 2.1 we get that $(\omega, t) \rightarrow r(\omega, h)(t)$ is measurable. Now fix $(\omega, t) \in \Omega \times T$. From an inequality of Pavel [13, (10.4), p. 129] we deduce that $h \rightarrow r(\omega, h)(t)$ is continuous. Hence $(\omega, t, h) \rightarrow$ $r(\omega, h)(t)$ is measurable, which in turn implies that

$$
(\omega, t, h) \rightarrow \widehat{F}(\omega, t, r(\omega, h)(t))
$$

is measurable. So, for every $x \in X,(\omega, t, h) \rightarrow d(x, \widehat{F}(\omega, t, r(\omega, h)(t)))$ is measurable. Since the distance function is continuous in $x$, we conclude that, for each $g \in L^{1}(T, X),(\omega, t, h) \rightarrow d(g(t), \widehat{F}(\omega, t, r(\omega, h)(t)))$ is measurable. From Fubini's theorem we get that

$$
(\omega, h) \rightarrow \int_{0}^{b} d(g(t), \widehat{F}(\omega, t, r(\omega, h)(t))) d t=d\left(g, S_{\widehat{F}(\omega, \cdot, r(\omega, h)(\cdot))}^{1}\right)
$$

is measurable. It then follows that the multifunction $Q: \Omega \times L^{1}(T, X) \rightarrow$ $P_{f c}\left(L^{1}(T, X)\right)$ defined by $Q(\omega, h)=S_{\widehat{F}(\omega, \cdot, r(\omega, h)(\cdot))}^{1}$ is measurable. Note that $Q(\omega, \cdot): B(\omega) \rightarrow P_{f c}(B(\omega))$. We claim that $Q(\omega, \cdot)$ is upper semicontinuous on $B(\omega)$ with the weak $L^{1}$-topology. Since $B(\omega)$ is weakly sequentially compact in $L^{1}(T, X)$ it suffices to show that $\operatorname{Gr} Q(\omega, \cdot)$ is sequentially closed in $B(\omega) \times B(\omega)$. Let $\left(h_{n}, g_{n}\right) \in \operatorname{Gr} Q(\omega, \cdot)$ such that $\left(h_{n}, g_{n}\right) \stackrel{w \times w}{\longrightarrow}(h, g)$ in $L^{1}(T, X) \times L^{1}(T, X)$. Since the semigroup $S(\omega, t)$ is compact for $t>0$, it follows from Vrabie [17] that $\overline{r(\omega, B(\omega))}$ is compact in $C(T, X)$. We may assume that $r\left(\omega, h_{n}\right) \rightarrow y$ in $C(T, X)$. Note that a mild solution is also an integral solution. So, from an inequality of Barbu [2, (2.4), p. 124], we get that $y=r(\omega, h)$. It then follows from hypothesis (ii) and theorem 3.1 of Papageorgiou [11] that $g(t) \in \widehat{F}(\omega, t, r(\omega, h)(t))$, that is, $g \in Q(\omega, h)$. So, $Q(\omega, \cdot)$ is upper semicontinuous from $B(\omega)$ into itself. Let $L(\omega)=\{f \in B(\omega): f \in Q(\omega, f)\}$. From Kakutani-Ky Fan fixed point theorem, we know that for all $\omega \in \Omega, L(\omega) \neq \varnothing$. Consider now the multifunction $M: \Omega \times L^{1}(T, X) \rightarrow L^{1}(T, X)$ defined by $M(\omega, f)=Q(\omega, f)-f$. Clearly $M$ is jointly measurable. Therefore $D=\{(\omega, f): 0 \in Q(\omega, f)-f\}$ belongs to $\Sigma \times B\left(L^{1}(T, X)\right)$. We note that $\operatorname{Gr} L=D \cap \operatorname{Gr} B(\cdot)$. Recalling that $\operatorname{Gr} B(\cdot) \in \Sigma \times B\left(L^{1}(T, X)\right)$, we conclude that $\operatorname{Gr} L \in \Sigma \times B\left(L^{1}(T, X)\right)$. Apply theorem 3 of Saint-Beuve [15] to get $v: \Omega \rightarrow L^{1}(T, X)$ measurable such that $v(\omega) \in L(\omega)$ for all $\omega \in \Omega$. Let $x(\omega, t)=r(\omega, v(\omega))(t)$. Clearly, $x(\omega, t)$ is the desired random mild solution of (3). 
Remark. The nonlinear version of Theorem 2.2 was proved in [1] under the assumption that $\omega \rightarrow \mathrm{Gr} A(\omega)$ is measurable from $\Omega$ into $P_{f}(X \times X)$, while in our case $A$ is a random operator. On the other hand the perturbation term $F$ in [1] satisfies the condition $|F(\omega, t, x)| \leq a(\omega, t)$, where $a(\cdot, \cdot)$ is jointly measurable and $a(\omega, \cdot) \in L^{1}\left(T, \mathbb{R}^{+}\right)$for all $\omega \in \Omega$, while in our result $F$ satisfies a more general growth condition.

\section{EXAMPLE}

Let $(\Omega, \Sigma, \mu)$ be a complete $\sigma$-finite measure space and $G$ a bounded domain in $\mathbb{R}^{n}$ with smooth boundary $\partial G$. Consider the following random, multivalued initial-boundary value problem on $\Omega \times T \times G$ :

$$
\begin{aligned}
\partial x(\omega, t, z) / \partial t & \in \Delta x(\omega, t, z)+F(\omega, t, z, x(\omega, t, z)) \\
x(\omega, t, z) & =0 \text { on } \Omega \times T \times \partial G, \\
x(\omega, 0, z) & =x_{0}(\omega, z) \text { on } \Omega \times\{0\} \times G .
\end{aligned}
$$

Here $F: \Omega \times T \times \mathbb{R}^{n} \times \mathbb{R} \rightarrow P_{f c}(\mathbb{R})$ is a multifunction such that

(i) for all $u \in \mathbb{R},(\omega, t, z) \rightarrow F(\omega, t, z, u)$ is measurable;

(ii) for all $(\omega, t, z) \in \Omega \times T \times \mathbb{R}^{n}, u \rightarrow F(\omega, t, z, u)$ is continuous;

(iii) $|F(\omega, t, z, u)| \leq a(\omega, t, z)$,

where $a(\cdot, \cdot, \cdot)$ is measurable and $a(\omega, \cdot, \cdot) \in L^{2}(T \times G)$. It is known (see [13, p. 141]) that if $A=\Delta$ with $D(A)=H_{0}^{1}(G) \cap H^{2}(G)$ then $A$ is a densely defined $m$-dissipative operator in $X=L^{2}(G)$ and the semigroup $S(t)$ generated by $A$ is compact for $t>0$. By hypotheses (i) and (ii) $(\omega, t, z, u) \rightarrow F(\omega, t, z, u)$ is measurable (see [10, Theorem 3.4]).

Now, let $\widehat{F}: \Omega \times T \times X \rightarrow P_{f c}(X)$ be defined by

$$
\widehat{F}(\omega, t, u)=S_{F(\omega, t, \cdot, u(\cdot))}^{2} .
$$

For $h \in X$ we have

$$
d(h, \widehat{F}(\omega, t, u))=\int_{G} d(h(z), F(\omega, t, z, u(z))) d z .
$$

From Fubini's theorem we get that $(\omega, t) \rightarrow d(h, \widehat{F}(\omega, t, u))$ is measurable, which implies that $(\omega, t) \rightarrow \widehat{F}(\omega, t, u)$ is measurable.

Let $B(\omega, t)=\left\{g \in X:\|g\|_{2} \leq\|a(\omega, t, \cdot)\|_{2}\right\}$. For every $g \in \widehat{F}(\omega, t, u)$ we have $g \in B(\omega, t)$. We assert that $u \rightarrow \widehat{F}(\omega, t, u)$ is u.s.c. from $X$ into $X_{w}$. Since $B(\omega, t)$ is $w$-compact and metrizable in the $w$-topology it is enough to show that $\operatorname{Gr} \hat{F}(\omega, t, \cdot)$ is sequentially closed in $X \times X_{w}$. To this end let $\left\{\left(u_{n}, g_{n}\right)\right\} \subset \mathrm{Gr} \widehat{F}(\omega, t, \cdot)$ such that $\left(u_{n}, g_{n}\right) \rightarrow(u, g)$ in $X \times X_{w}$. We have $g_{n}(z) \in F\left(\omega, t, z, u_{n}(z)\right)$. By hypothesis (ii) and [11, Theorem 3.1] we conclude that $g(z) \in F(\omega, t, z, u(z))$. Hence $g \in \widehat{F}(\omega, t, u)$ and so $u \rightarrow \widehat{F}(\omega, t, u)$ is u.s.c. from $X$ into $X_{w}$. Combining hypothesis (ii) and [11, 
Theorem 4.1] we get that $u \rightarrow \widehat{F}(\omega, t, u)$ is l.s.c. From [10, Theorem 3.4] we get that $(\omega, t, u) \rightarrow \widehat{F}(\omega, t, u)$ is measurable. Now, we can easily check that

$$
|\widehat{F}(\omega, t, u)| \leq \hat{a}(\omega, t),
$$

where $\hat{a}(\omega, t)=\|a(\omega, t, \cdot)\|_{2}$. Define $v_{0}: \Omega \rightarrow X$ by $v_{0}(\omega)(\cdot)=x_{0}(\omega, \cdot)$ and rewrite (4) as the following abstract evolution equation

$$
\begin{aligned}
\dot{v}(\omega, t) & =A v(\omega, t)+\widehat{F}(\omega, t, v(\omega, t)) \\
v(\omega, 0) & =v_{0}(\omega) .
\end{aligned}
$$

Then applying Theorem 2.2 we deduce that (5) has a random mild solution $v: \Omega \times T \rightarrow X$. Set $x(\omega, t, z)=v(\omega, t)(z)$. This is the desired random distributional solution of (4).

\section{REFERENCES}

1. E. Avgerinos and N. S. Papageorgiou, Random nonlinear evolution inclusions in reflexive Banach spaces, Proc. Amer. Math. Soc. 104 (1988), 293-299.

2. V. Barbu, Nonlinear semigroups and differential equations in Banach spaces, Noordhoff, Leyden, 1976.

3. G. Becus, Random generalized solutions to the heat equation, J. Math. Anal. Appl. 90 (1977), 93-102.

4. A. Bharucha-Reid, Random integral equations, Academic Press, New York, 1972.

5. S. Itoh, Random fixed point theorems with an application to random differential equations in Banach spaces, J. Math. Anal. Appl. 67 (1979), 261-273.

6. __ Random differential equations associated with accretive operators, J. Differential Equations 31 (1979), 139-154.

7. J. Kampé de Feriet, Random solutions of partial differential equations (Proc. 3rd Berkeley Sympos. Math. Stat. and Probab.), vol. III, Univ. of California Press, Berkeley, 1956, pp. 199-208.

8. D. Kravvaritis and N. Stavrakakis, Perturbations of maximal monotone random operators, Linear Alg. Appl. 84 (1986), 301-310.

9. N. S. Papageorgiou, Random differential inclusions in Banach spaces, J. Differential Equations 65 (1986), 287-303.

10. __ On measurable multifunctions with applications to random multivalued equations, Math. Japonica 32 (1987), 701-727.

11. Convergence theorems for Banach spaces valued integrable multifunctions, Internat. J. Math. Math. Sci. 10 (1987), 433-442.

12. N. Pavel, Differential equations, flow invariance and applications, Research Notes in Math., vol. 113, Pitman, London, 1984.

13. __ Nonlinear evolution operators and semigroups, Lecture Notes in Math., vol. 1260, Springer-Verlag, Berlin and New York, 1987.

14. A. Pazy, A class of semi-linear equations of evolution, Israel J. Math. 20 (1975), 23-36.

15. M.-F. Saint-Beuve, On the extension of von Neumann-Aumann's theorem, J. Funct. Anal. 17 (1974), 112-129.

16. T. Soong, Random differential equations in science and engineering, Academic Press, New York, 1973. 
17. I. Vrabie, Nonlinear evolution equations: Existence via compactness (H. Brezis, M. Grandall, and F. Kappel, eds.), Pitman, New York, 1986, pp. 242-248.

18. _ The nonlinear version of Pazy's local existence theorem, Israel J. Math. 32 (1979), 221-235.

Department of Mathematics, National Technical University of Athens, Zografou Campus, Zografou, 15773 Athens, Greece 